\title{
Is anchorage reinforcement with implants effective in orthodontics?
}

\section{Is anchorage reinforcement using implants or other surgical methods as effective as conventional techniques when people undergo orthodontic treatment?}

\author{
Skeggs RM, Benson PE, Dyer F. \\ Reinforcement of anchorage during orthodontic brace treatment \\ with implants or other surgical methods. Cochrane Database Syst \\ Rev 2007; issue 3
}

Data sources The Cochrane Oral Health Group's Trials Register, Cochrane Central Register of Controlled Trials (CENTRAL), Medline and Embase were searched. No language restrictions were applied. Authors were identified and contacted to identify unpublished trials.

Study selection Studies selected were randomised clinical trials (RCT) or quasi-RCT involving surgically assisted means of anchorage reinforcement in orthodontic patients.

Data extraction and synthesis Data extraction was performed by two review authors working independently using a previously piloted data collection form. Data were entered into RevMan (The Nordic Cochrane Centre. Copenhagen, Denmark); planned analysis of mean differences and $95 \%$ confidence intervals $(\mathrm{Cl})$ for continuous outcomes, and risk ratios (RR) and $95 \% \mathrm{Cl}$ for dichotomous outcomes. Pooling of data and meta-analysis were not performed because there were too few similar studies.

Results To date, few trials have been carried out in this field and there are insufficient data of adequate quality in the literature to meet the objectives of the review. The review authors were only able to find one study that assessed the use of surgical anchorage reinforcement systems. This trial examined 51 patients with absolute anchorage requirements treated in two centres. Patients were randomly allocated to receive either headgear or a midpalatal osseo-integrated implant. Anchorage loss was measured cephalometrically by mesial movement of dental and skeletal reference points between the start of treatment and the end of anchorage reinforcement. All skeletal and dental points moved mesially more in the headgear group than the implant group. Results showed significant differences for mesial movement of the maxillary molar in both groups. The mean change in the implant group was $1.5 \mathrm{~mm}$ [standard deviation (SD), 2.6; 95\% Cl, 0.4-2.7] and for the headgear group was $3.0 \mathrm{~mm}$ (SD, 3.4; 95\% Cl, 1.6-4.5). The trial was designed to test a clinically significant difference of $2 \mathrm{~mm}$, so the result was not statistically significant, but the authors conclude that midpalatal implants do effectively reinforce anchorage and are an acceptable alternative to headgear in absolute anchorage cases.
Conclusions There is limited evidence that osseo-integrated palatal implants are an acceptable means of reinforcing anchorage. The review authors were unable to identify trials addressing the secondary objectives of the review relating to patient acceptance, discomfort and failure rates. In view of the fact that this is a dynamic area of orthodontic practice, there is a need for high-quality RCT. There are financial restrictions in running trials of this nature but it would be in the interest of implant manufacturers to fund such quality, independently conducted trials of their products.

\section{Commentary}

Newton's third law and the biological sensitivity of bone to applied forces have always confounded and challenged the orthodontist in clinical practice. To add to this, the need for patient compliance and co-operation in conventional anchorage reinforcement, where extra oral traction and elastics are used, confounds the issue further. As the whole issue of absolute anchorage gathers momentum, a Cochrane review is bound to be received with enthusiasm and interest by the profession.

A fair amount of work has been published that deals with both the basic science and the clinical usage of adjuncts in anchorage reinforcement, which necessitates the review. The question that will eventually need to be answered is how we interpret the available evidence, within the narrow confines of the stringent evidence-based protocol or within the open vistas of basic research, and how we extrapolate the facts into clinical practice.

The present review defined its objectives very clearly, comparing the surgical interventions in anchorage reinforcement with conventional methods, with a secondary aim of examining patient acceptability and failure. In terms of the evidence-based process the review is critical, objective and worthy of praise for its well-defined parameters, search strategy, inclusion and exclusion criteria. The paucity of sufficiently high-quality data prevented the authors pooling data and performing a meta-analysis. The derivation of a conclusion based on the one study meeting the inclusion criteria would compromise the outcome of the review but the suggestion that there is little evidence to support the use of surgical anchorage systems over conventional means of anchorage reinforcement seems misplaced.

The authors' conclusion that there is limited evidence to support the use of the midpalatal osseo-integrated implants as reinforcement of anchorage is absolutely valid and correct. The osseo-integrated implant comes with its own set of issues related to invasive intervention, time lag before the implant can be loaded, and the fact that the biomechanics of a midpalatal implant may not be ideal in reinforcing anchorage. The very fact that the process of osseo-integration would add at least another 3-4 months to the overall treatment time is in itself a limiting feature. So should the search for evidence move back into the realm of basic science? Some facts are already scripted: osseo-integration is not really required in terms of orthodontic force
Address for correspondence: Luisa M Fernandez Mauleffinch, Cochrane Ora Health Group, MANDEC, School of Dentistry, University of Manchester, Higher Cambridge Street, Manchester M15 6FH, UK.

E-mail: luisa.fernandez@manchester.ac.uk 
application; bone responds very well to lateral loading, improving the retention of any implant; titanium alloys with excellent biocompatibility are available; and the ease with which micro-implants can be used in a wide range of clinical applications provides a new perspective to absolute anchorage.

The whole issue has been well-researched in this review. RCT are few, but it is not difficult to arrive at conclusions based on the facts available so far. Anchorage loss is well-researched and documented, and so is the increasing body of work on the clinical applications of micro-implants providing a fixed point of force application, not taxing the periodontal tissues for both orthodontic and orthopaedic change. The Holy Grail is in our sights.

The research question for the systematic review is well defined and looks to review the entire spectrum of implants and surgical methods of reinforcing anchorage in comparison to conventional means. The authors conclude however that there is limited evidence on the value of osseo-integrated implants in reinforcing anchorage. Would this exclude all other means of anchorage reinforcement such as micro implants and plates as well? The present review, while clearly outlining the objective of comparing surgically reinforced anchorage systems with conventional means of anchorage reinforcement, has been limited by the paucity of good evidence in the form of RCTs. The authors have done a comprehensive literature search and defined stringent criteria. In terms of evidencebased methods it is an impeccable study. It is evident however that the paucity of good evidence would limit the authors in deriving a clear conclusion. Rather than limiting the outcome of an excellent evidence based effort, it would better to accept the limitations in terms of evidence and defer the conclusions till a greater body of evidence comes to light.

\section{Anmol S Kalha}

Department of Orthodontics, Institute Of Dental Studies and Technology, Delhi, India

Evidence-Based Dentistry (2008) 9, 13-14. doi:10.1038/sj.ebd.6400564 